\title{
El papel del Observatorio de Salud Mental y los criterios de calidad asistencial de la AEN en la evaluación y planifi- cación asistencial. Recursos de profesionales en CSM y de dispositivos de atención a pacientes con TMG en España.
}

The Mental Health Observatory's role and AEN's assistance quality criteria in healthcare evaluation and planning. Professional resources in Mental Health Services and in Severe Mental Health Disorders attention units in Spain.

\author{
Juan Fco. Jiménez Estévez ${ }^{a}$ \\ ${ }^{a}$ Psicólogo clínico. Unidad de Rehabilitación de Salud Mental. Hospital Universitario San Cecilio de Gra- \\ nada. Responsable del Observatorio de Salud Mental de la AEN.
}

Correspondencia: Juan Fco. Jiménez Estévez (juanf.jimenez.sspa@ juntadeandalucia.es)

\begin{abstract}
RESUMEN: En el presente trabajo se presentan algunos datos preliminares sobre recursos profesionales en centros de salud mental de adultos (CSM) y de diversos dispositivos para pacientes con trastorno mental grave (TMG) recogidos por el Observatorio de Salud Mental de la AEN 2010 en cada una de las comunidades autónomas. Los datos mostrados en forma de tasas de profesionales y de plazas en los dispositivos asistenciales permiten analizarlos en relación con las estimaciones de criterios de calidad que , para estos profesionales y dispositivos, han sido establecidos por la propia AEN. Los datos presentados permiten afirmar que la dotación de recursos profesionales en centros de salud mental de adultos y de plazas en dispositivos asistenciales para pacientes mentales graves es, en general, heterogénea e insuficiente para alcanzar unos indicadores mínimos de calidad. El Observatorio de Salud Mental de la AEN junto a la estimación de criterios de calidad de la AEN son dos instrumentos que en combinación permiten realizar un seguimiento y evaluación de la red asistencial del Sistema Nacional de Salud.
\end{abstract}

PALABRAS CLAVE: observatorio, salud mental, recursos humanos, red asistencial, indicadores de calidad
ABSTRACT: In the following paper some preliminary data is portrayed about the professional resources in adult mental health services (CSM) and the several services assisting severe mental health patients (TMG) collected by the AEN's Mental Health Observatory in 2010 in each autonomous region. Data displayed in the form of professional rates and positions in the assistance services allows being analised in connection with the estimation of quality criteria which, for these professionals and services, has been established by the same AEN. Presented data supports the statement that professional resources supply in mental health services for severe mental health patients is, generally, heterogeneous and insufficient in reaching minimum quality indicators. Both AEN's Mental Health Observatory, along with the AEN quality criteria assessment, are two mechanisms which in combination allow carrying out a follow up and evaluation of the assisting network in the National Health System.

KEY WORDS: observatory, mental health, human resources, assisting network, quality indicator. 


\section{Introducción}

El Observatorio de Salud Mental es un proyecto estratégico que la Asociación Española de Neuropsiquiatría (AEN) decidió poner en marcha hace casi ya una década, dada la limitación, precariedad y fragmentación de sistemas de información acerca de los servicios asistenciales a la salud mental en el Sistema Nacional de Salud. La pertinencia de este proyecto partía de la necesidad de conocer el desarrollo y la implementación del modelo de asistencia comunitario impulsado por los diferentes planes de reforma psiquiátrica nacionales y autonómicos. Para ello puso en marcha un grupo de trabajo constituido por representantes de todas las comunidades autónomas con la responsabilidad de elaborar y revisar la encuesta, base del observatorio, y realizar la recogida de datos de los propios servicios autonómicos de salud mental. Este servicio se ha concebido como un instrumento permanente de modo que viene recogiendo de forma periódica y sistemática, información sobre la organización de la asistencia a la salud mental en el conjunto del estado español (1-5). La última edición ha sido presentada este año $(4,5)$ y elaborada durante 2010 a partir de los datos facilitados por los diversos servicios autonómicos de salud mental a fecha de 31 de diciembre de 2009.

Como se ha venido reflejando en las diferentes ediciones del Observatorio de Salud Mental de la Asociación Española de Neuropsiquiatría y en la propia Estrategia de Salud Mental del Sistema Nacional de Salud (6), la implantación del modelo comunitario en España ha sido hetérogéneo y desigual en las diferentes comunidades autónomas. Aunque de un modo generalizado se han diversificado en el conjunto del estado las redes asistenciales, los dispositivos y prestaciones, junto a un aumento sustancial de los recursos humanos dedicados a la atención a la salud mental, éstos se han desarrollado de una manera desigual, fruto de situaciones de partida, políticas y prioridades sanitarias diferentes.

El Observatorio de Salud Mental de la AEN en su nueva edición correspondiente a 2010 nos ofrece un extenso conjunto de datos acerca de la asistencia a la salud mental en en cada una de las comunidades autónomas. Estos datos no sólo nos permiten conocer la realidad de la asistencia a la salud en España, sino que nos permiten conocer su evolución y la posibilidad de hacer valoraciones de esta realidad en función de indicadores de calidad asistencial, como la misma Estrategia de Salud Mental establece como objetivo.

La estimación de recursos necesarios para una atención de calidad a los problemas de salud mental es una tarea compleja que debería poder derivarse de los mejores datos acerca de la efectividad y eficiencia sobre la organización de servicios, dispositivos e intervenciones terapéuticas y profesionales, en función de los estudios de prevalencia geográfica de las distintas patologías mentales. Sin embargo, la escasez de datos y las limitaciones de los mismos obliga a recurrir a reco- 
INFORMES

mendaciones y consensos de expertos. En ausencia de criterios más objetivos, con ellos podemos establecer una hoja de ruta con unos criterios mínimos que permitan orientarnos a una red asistencial en consonancia con los objetivos y estrategias definidas en el marco político-sanitario de la Declaración de Helsinki sobre Salud Mental (7) para el espacio europeo, o la Estrategia de Salud Mental en el Sistema Nacional de Salud $(6,8)$, en el ámbito del sistema sanitario español, que vienen inspirando la redacción y los compromisos de los planes de salud mental de las diferentes comunidades autónomas.

En general, desde el Observatorio de Salud Mental de la AEN, venimos observando un crecimiento lento y desigual, tanto de recursos humanos como de dispositivos asistenciales, en el conjunto de las comunidades autónomas, que parece responder más a puntos de partida histórico-sanitarios y a las disponibilidades presupuestarias que a una auténtica planificación en función de criterios poblacionales.

La elaboración y asunción de estrategias de salud mental, planes de salud mental, guías de práctica clínica o procesos asistenciales han ido perfilando en los últimos años el establecimiento de unos modelos asistenciales con unas características de calidad que requieren de una dotación de recursos mínimos para poder alcanzar sus objetivos y no quedar reducidos a una mera declaración de principios. Es evidente que si se pretende conseguir una atención a la salud mental con criterios de calidad, además de una adecuada planificación se requiere una dotación de recursos proporcional en relación con las necesidades asistenciales, los objetivos estratégicos y la población a atender.

Por ello la Asociación Española de Neuropsiquiatría, partiendo de estos presupuestos y siendo consciente de que los recursos no son ilimitados ya estableció en el documento "Hacia una atención comunitaria de Salud Mental de calidad" (9), una estimación de recursos mínimos para una atención de calidad en salud mental en el conjunto del estado español. Estas estimaciones han sido realizadas a partir de diversos estudios epidemiológicos y de documentos y grupos de trabajo tanto nacionales como internacionales.

El Observatorio de Salud Mental y los criterios de calidad asistencial vienen a suponer dos importantes instrumentos complementarios desarrollados por la AEN en su estrategia de servicio para la mejora de la asistencia a la salud mental en España. El presente estudio pretende mostrar una aproximación a las posibilidades que nos brinda esta combinación de recursos y su utilidad para la evaluación y planificación de políticas asistenciales. Para ello se revisarán los recursos profesionales (psiquiatras, psicólogos clínicos y enfermería) de los centros de salud mental de adultos y diversos dispositivos para la atención a pacientes con trastorno mental grave. 


\section{Recursos profesionales de la red de salud mental}

El Observatorio de Salud Mental de la AEN proporciona información acerca de la dotación de recursos profesionales en los diversos dispositivos de atención a la salud mental en cada una de las comunidades autónomas. En este trabajo vamos a ver la dotación de plazas de psiquiatras, psicólogos clínicos y enfermería en los centros de salud mental de adultos (CSM) de las diferentes comunidades autónomas.

\section{Psiquiatras en Centros de Salud de Adultos}

En la tabla1 podemos ver la dotación de psiquiatras por comunidades autónomas y en el conjunto del Estado. En ella podemos observar el número de psiquiatras en cada una de las comunidades autónomas y su tasa por cada 100.000 habitantes. También podemos observar la tasa y el número de psiquiatras que faltan

Tabla 1

Psiquiatras en centros de salud mental de adultos. Número total por comunidades, tasas por 100.000 habitantes y número y tasas de psiquiatras que faltan según criterio de calidad de la AEN.

\begin{tabular}{|c|c|c|c|c|}
\hline Comunidad Autónoma & Psiquiatras & $\begin{array}{c}\text { Tasa } x \\
100.000 \text { h. }\end{array}$ & $\begin{array}{c}\text { Faltan } x \\
100.000 \text { h. }\end{array}$ & Faltan $n^{o}$ \\
\hline Andalucía & 296 & 3,57 & 1,43 & 119 \\
\hline Aragón & 46 & 3,42 & 1,58 & 21 \\
\hline Asturias & 56,4 & 5,2 & $-0,2$ & -2 \\
\hline C. de Madrid & 257 & 4,02 & 0,98 & 63 \\
\hline C. de Murcia & 50 & 3,46 & 1,54 & 22 \\
\hline C. Valenciana & 184 & 3,61 & 1,39 & 71 \\
\hline Cantabria & 21 & 3,56 & 1,44 & 8 \\
\hline Castilla la Mancha & 86 & 4,13 & 0,87 & 18 \\
\hline Castilla León & 90 & 3,51 & 1,49 & 38 \\
\hline Extremadura & 37 & 3,36 & 1,64 & 18 \\
\hline Galicia & 64,2 & 2,3 & 2,7 & 75 \\
\hline Islas Baleares & 27 & 2,46 & 2,54 & 28 \\
\hline Islas Canarias & 55 & 2,61 & 2,39 & 50 \\
\hline La Rioja & 10 & 3,11 & 1,89 & 6 \\
\hline Navarra & 27,5 & 4,36 & 0,64 & 4 \\
\hline País Vasco & 108,5 & 4,99 & 0,01 & 0 \\
\hline Total & $1.074,68$ & & \multicolumn{2}{|c|}{ Faltan total } \\
\hline Promedio & 63,22 & 3,39 & \multicolumn{2}{|c|}{539} \\
\hline Desv. estandar & 85,91 & 0,81 & & \\
\hline
\end{tabular}

* No se dispone de datos de Cataluña 
en cada comunidad autónoma según el criterio de calidad de la AEN para este tipo de dispositivos. No disponemos de datos referidos a Cataluña. La AEN estima en 5 psiquiatras la dotación mínima para una prestación asistencial de calidad de los centros de salud mental de adultos.

En el conjunto del Estado 1075 psiquiatras prestan su actividad asistencial en centros de salud mental de adultos, lo que representa un total de 3.4 psiquiatras por cada 100.000 habitantes. Dado que la AEN estima en un mínimo de 5 la dotación mínima de psiquiatras para centros de salud mental de adultos, ello implica que sólo está dotado el $66.6 \%$ de los recursos necesarios y que, por tanto, sería necesaria la contratación de 539 nuevos psiquiatras para alcanzar los 1614 necesarios para cumplir el indicador de calidad.

Si observamos la dotación de psiquiatras por comunidades autónomas, únicamente Asturias, con 5.20 psiquiatras por cada 100.000 habitantes y País Vasco, con 4.99 psiquiatras por cada 100.000 habitantes, alcanzan dicho criterio de calidad. Galicia, las Islas Baleares, las Islas Canarias, La Rioja o Extremadura, no sólo no alcanzan el criterio de calidad de la AEN, sino que se encuentran por debajo de la media de las comunidades autónomas en cuanto a dotación de psiquiatras. Galicia y las Islas Baleares incluso una desviación típica por debajo de una media ya de por sí baja para el conjunto del Estado.

En la figura 1 podemos ver la representación gráfica de la distribución de tasas por comunidades autónomas y en relación al criterio de calidad de la AEN.

Aunque la mayoría de las administraciones autonómicas, y particularmente la murciana, han ido aumentando la dotación de psiquiatras en los últimos años este incremento es aún insuficiente para una dotación profesional que garantice, según

Figura 1

Psiquiatras en centros de salud mental de adultos. Tasas por cada 100.000 habitantes

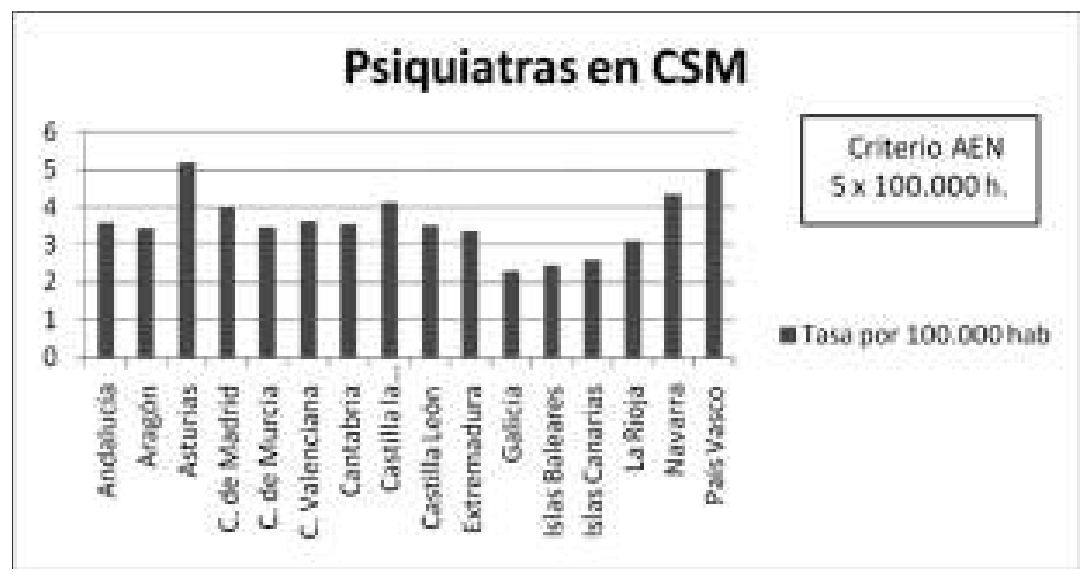


los criterios de calidad, las necesidades asistenciales de la población. En la figura 2 podemos observar la proporción y el número de psiquiatras existentes y faltantes para cada una de las comunidades autónomas. Podemos observar en la figura como las comunidades de Galicia e Islas Baleares ni siquiera cubren el $50 \%$ de las necesidades estimadas por la AEN.

Figura 2

Relación entre plazas de psiquiatría existentes en centros de salud mental de adultos y plazas que faltarían según el criterio de calidad de la AEN

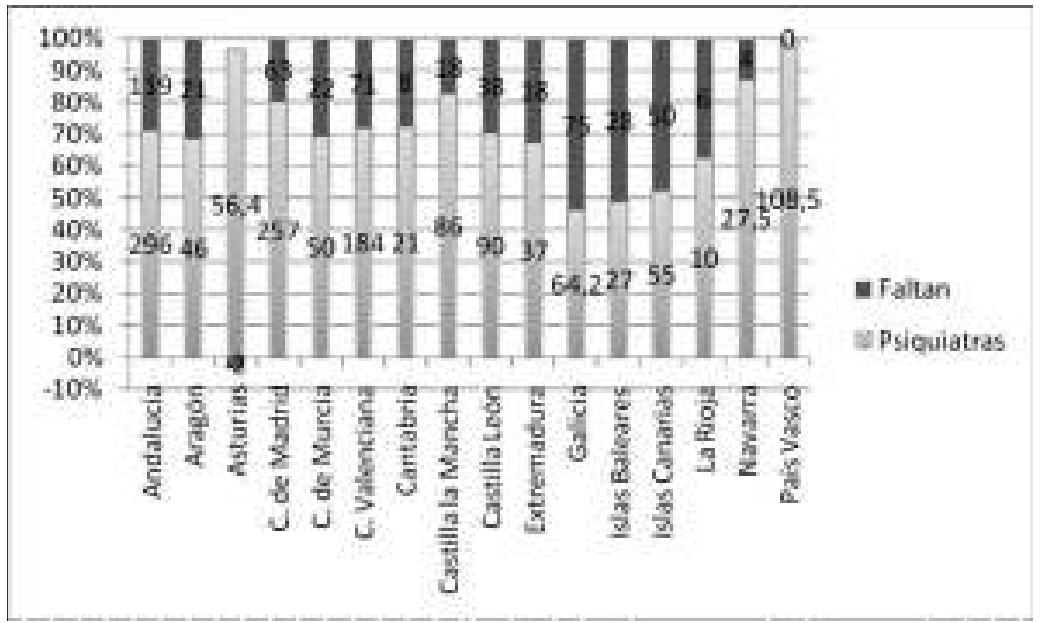

\section{Psicólogos clínicos en Centros de Salud Mental de Adultos}

En la tabla 2 podemos ver la dotación de psicólogos por comunidades autónomas y en el conjunto del Estado. En ella podemos observar el número de psicólogos en cada una de las comunidades autónomas y su tasa por cada 100.000 habitantes. También podemos observar la tasa y el número de psicólogos que faltan en cada comunidad autónoma según el criterio de calidad de la AEN para este tipo de dispositivos. No disponemos de datos referidos a Cataluña. La AEN estima en 4 psicólogos clínicos la dotación mínima para una prestación asistencial de calidad de los centros de salud mental de adultos.

En el conjunto del Estado 564 psicólogos clínicos prestan su actividad asistencial en centros de salud mental de adultos, lo que representa un total de 2 psicólogos por cada 100.000 habitantes. Dado que la AEN estima en un mínimo de 4 la dotación mínima de psicólogos clínicos para centros de salud mental de adultos, ello implica que sólo está dotado el $41.7 \%$ de los recursos necesarios y que, por tanto, sería necesaria la contratación de 790 nuevos psicólogos clínicos para llegar a los 1355 necesarios para alcanzar este índice de calidad. 
Tabla 2

Psicólogos clínicos en centros de salud mental de adultos. Número total por comunidades, tasas por 100.000 habitantes y número y tasas de psicólogos que faltan según criterio de calidad de la AEN.

\begin{tabular}{|c|c|c|c|c|}
\hline Comunidad Autónoma & Psicólogos & $\begin{array}{c}\text { Tasa } x \\
100.000 \mathrm{~h} .\end{array}$ & $\begin{array}{c}\text { Faltan } x \\
100.000 \text { h. }\end{array}$ & Faltan $n^{o}$ \\
\hline Andalucía & 144 & 1,73 & 2,27 & 189 \\
\hline Aragón & 25 & 1,86 & 2,14 & 29 \\
\hline Asturias & 22 & 2,06 & 1,94 & 21 \\
\hline C. de Madrid & 120 & 1,88 & 2,12 & 135 \\
\hline C. de Murcia & 39 & 2,7 & 1,3 & 19 \\
\hline C. Valenciana & 97 & 1,9 & 2,1 & 107 \\
\hline Cantabria & 14 & 2,38 & 1,62 & 10 \\
\hline Castilla la Mancha & 67 & 3,22 & 0,78 & 16 \\
\hline Castilla León & 48 & 1,87 & 2,13 & 55 \\
\hline Extremadura & 29 & 2,63 & 1,37 & 15 \\
\hline Galicia & 34 & 1,2 & 2,8 & 78 \\
\hline Islas Baleares & 23 & 2,1 & 1,9 & 21 \\
\hline Islas Canarias & 49 & 2,33 & 1,67 & 35 \\
\hline La Rioja & 8 & 2,49 & 1,51 & 5 \\
\hline Navarra & 18 & 2,85 & 1,15 & 7 \\
\hline País Vasco & 39 & 1,78 & 2,22 & 48 \\
\hline Total & 563,94 & & \multicolumn{2}{|c|}{ Faltan total } \\
\hline Promedio & 33,17 & 2,06 & & 790 \\
\hline Desv. estandar & 41,16 & 0,51 & & \\
\hline
\end{tabular}

* No se dispone de datos de Cataluña

Si observamos la dotación de psicólogos clínicos por comunidades autónomas, ninguna comunidad autónoma cumple el criterio de calidad de la AEN. La comunidad que más se acerca a éste es Castilla la Mancha, mientras que Galicia, Andalucía, País Vasco, Aragón, Castilla y León, la Comunidad de Madrid o la Comunidad de Valencia, no alcanzan la media del Estado. Galicia se encuentra incluso a una desviación típica por debajo de una ya baja media estatal situada en el $50 \%$ de las necesidades estimadas.

En la figura 3 podemos ver la representación gráfica de la distribución de tasas por comunidades autónomas y en relación al criterio de calidad de la AEN.

Como podemos observar en la figura 4 , todas las comunidades necesitan ampliar la dotación de psicólogos clínicos en proporción y número significativos para cumplir el criterio de calidad de la AEN. Aunque en los últimos años algunas comunidades han aumentado su plantilla de psicólogos clínicos de modo conside- 
Figura 3

Psicólogos clínicos en centros de salud mental de adultos. Tasas por cada 100.000 habitantes

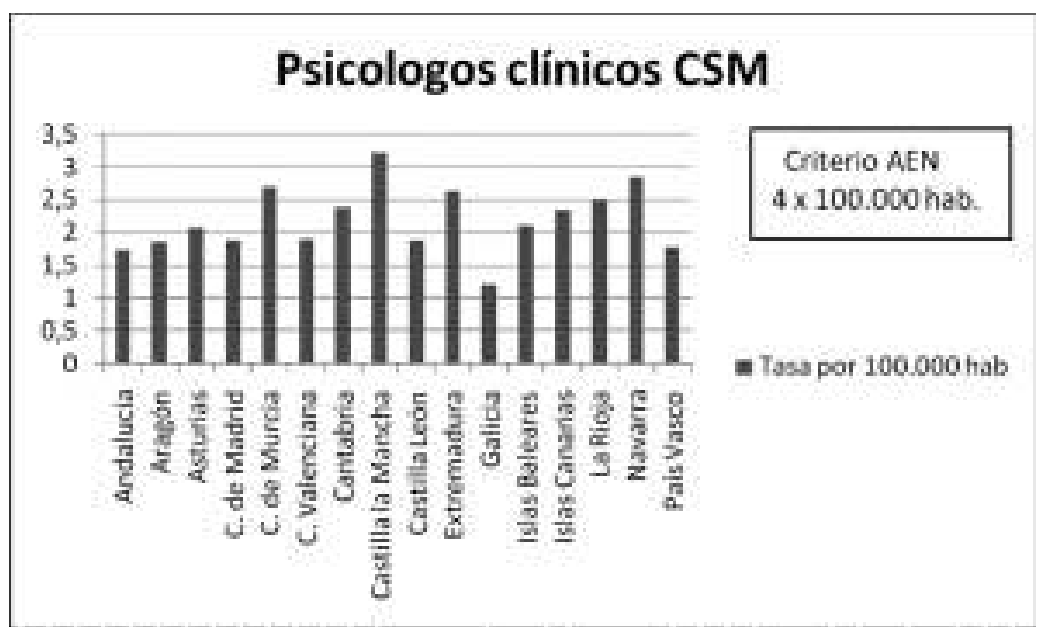

rable como Extremadura, Castilla la Mancha, Asturias y, sobre todo, Murcia, dadas las carencias de las que se parte aún son necesarios más profesionales. Galicia, Andalucía, País Vasco, Aragón, Castilla y León, la Comunidad de Madrid o la Comunidad de Valencia, no tienen cubiertas ni siquiera el 50\% de las necesidades de psicólogos en centros de salud mental para una mínima atención de calidad de su población asistencial.

Figura 4

Relación entre plazas de psicología clínica existentes en centros de salud mental de adultos y plazas que faltarían según el criterio de calidad de la AEN

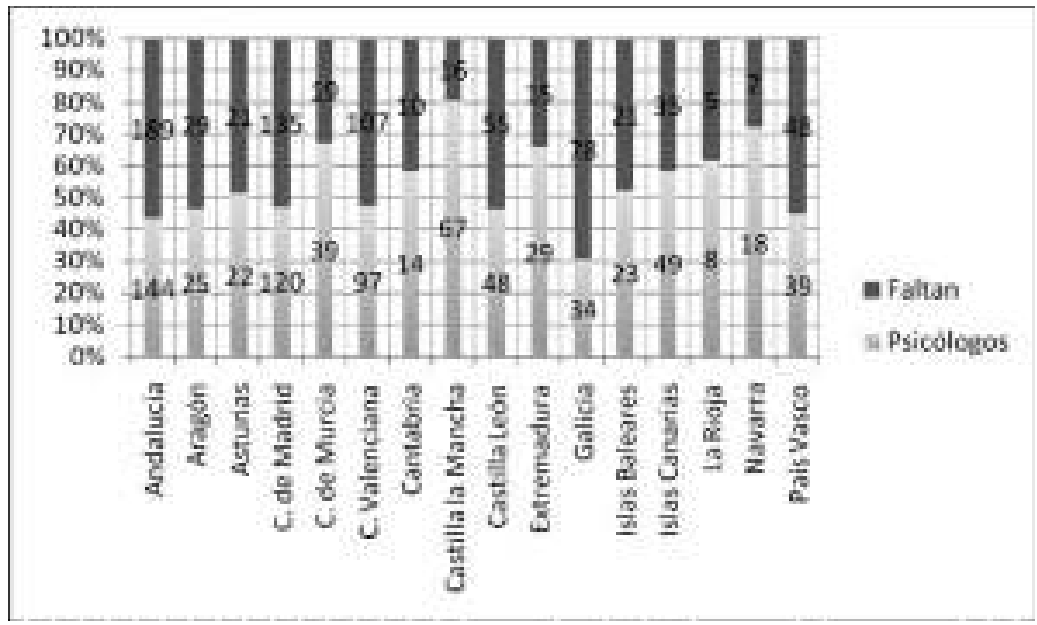




\section{Enfermería en Centros de Salud Mental de Adultos}

En la tabla 3 podemos ver la dotación de enfermería por comunidades autónomas y en el conjunto del Estado. En ella podemos observar el número de enfermeras en cada una de las comunidades autónomas y su tasa por cada 100.000 habitantes. También podemos observar la tasa y el número de enfermeras que faltan en cada comunidad autónoma según el criterio de calidad de la AEN para este tipo de dispositivos. No disponemos de datos referidos a Cataluña. La AEN estima en 3 enfermeras la dotación mínima para una prestación asistencial de calidad de los centros de salud mental de adultos.

En el conjunto del Estado 530 enfermeras prestan su actividad asistencial en centros de salud mental de adultos, lo que representa un total de 1,8 enfermeras por cada 100.000 habitantes. Dado que la AEN estima en un mínimo de 3 la dotación mínima de enfermería para centros de salud mental de adultos, ello implica que

Tabla 3

Enfermería en centros de salud mental de adultos. Número total por comunidades, tasas por 100.000 habitantes y número y tasas de enfermería que faltan según criterio de calidad de la AEN.

\begin{tabular}{|c|c|c|c|c|}
\hline Comunidad Autónoma & $\begin{array}{l}\text { Tasa } x \\
\text { Enfermería }\end{array}$ & $\begin{array}{l}\text { Faltan } x \\
100.000 h .\end{array}$ & $100.000 \mathrm{~h}$. & Faltan $n^{o}$ \\
\hline Andalucía & 156 & 1,88 & 1,12 & 93 \\
\hline Aragón & 20 & 1,49 & 1,51 & 20 \\
\hline Asturias & 30 & 2,76 & 0,24 & 3 \\
\hline C. de Madrid & 100 & 1,57 & 1,43 & 91 \\
\hline C. de Murcia & 35 & 2,42 & 0,58 & 8 \\
\hline C. Valenciana & 80 & 1,57 & 1,43 & 73 \\
\hline Cantabria & 7 & 1,19 & 1,81 & 11 \\
\hline Castilla la Mancha & 38 & 1,83 & 1,17 & 24 \\
\hline Castilla León & 42 & 1,64 & 1,36 & 35 \\
\hline Extremadura & 23 & 2,09 & 0,91 & 10 \\
\hline Galicia & 24 & 0,86 & 2,14 & 60 \\
\hline Islas Baleares & 16 & 1,46 & 1,54 & 17 \\
\hline Islas Canarias & 47 & 2,23 & 0,77 & 16 \\
\hline La Rioja & 9 & 2,8 & 0,2 & 1 \\
\hline Navarra & 14 & 2,22 & 0,78 & 5 \\
\hline País Vasco & 72,7 & 3,35 & $-0,35$ & -8 \\
\hline Total & 530,25 & & \multicolumn{2}{|c|}{ Faltan total } \\
\hline Promedio & 31,19 & 1,84 & \multicolumn{2}{|c|}{459} \\
\hline Desv. Estandar & 40,85 & 0,64 & & \\
\hline
\end{tabular}

* No se dispone de datos de Cataluña 
sólo está dotado el 53,6\% de los recursos necesarios y que, por tanto, sería necesaria la contratación de 459 nuevos enfermeros para llegar a los 989 necesarios para alcanzar este índice de calidad.

Si observamos la dotación de profesionales de enfermería por comunidades autónomas, únicamente el País Vasco cumple el criterio de calidad de la AEN. Se acercan al mismo La Rioja y Asturias. Mientras, Galicia, Cantabria, Islas Baleares, Aragón, la Comunidad de Madrid o la Comunidad de Valencia, Castilla y León y Andalucía no alcanzan la media del Estado. Galicia y Cantabria se encuentran incluso a una desviación típica de la ya de por sí baja media del Estado situada casi en la mitad de las necesidades requeridas.

En la figura 5 podemos ver la representación gráfica de la distribución de tasas por comunidades autónomas y en relación al criterio de calidad de la AEN.

Figura 5

Enfermería en centros de salud mental de adultos. Tasas por cada 100.000 habitantes

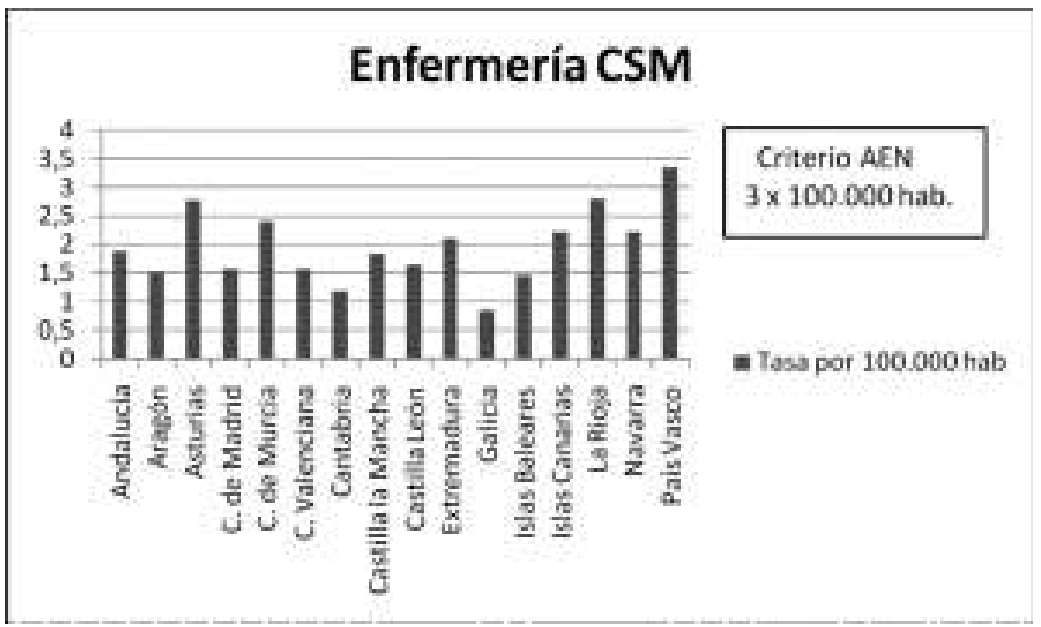

Como podemos observar también en la figura 6, casi todas las comunidades salvo el País Vasco necesitan ampliar la dotación de enfermería en proporción y número significativos para cumplir el criterio de calidad de la AEN. En los últimos años Asturias y, sobre todo, Murcia, han aumentado la dotación de enfermería en los centros de salud mental de adultos de forma importante. La Rioja, Asturias y Murcia sólo necesitarían 1, 3 y 8 nuevas plazas respectivamente para alcanzar el indicador de calidad de 3 enfermeras por cada 100.000 habitantes. Sin embargo Galicia, Cantabria y las Islas Baleares no tienen cubiertas ni siquiera el $50 \%$ de las necesidades de enfermería en centros de salud mental para una atención de calidad de su población asistencial. 


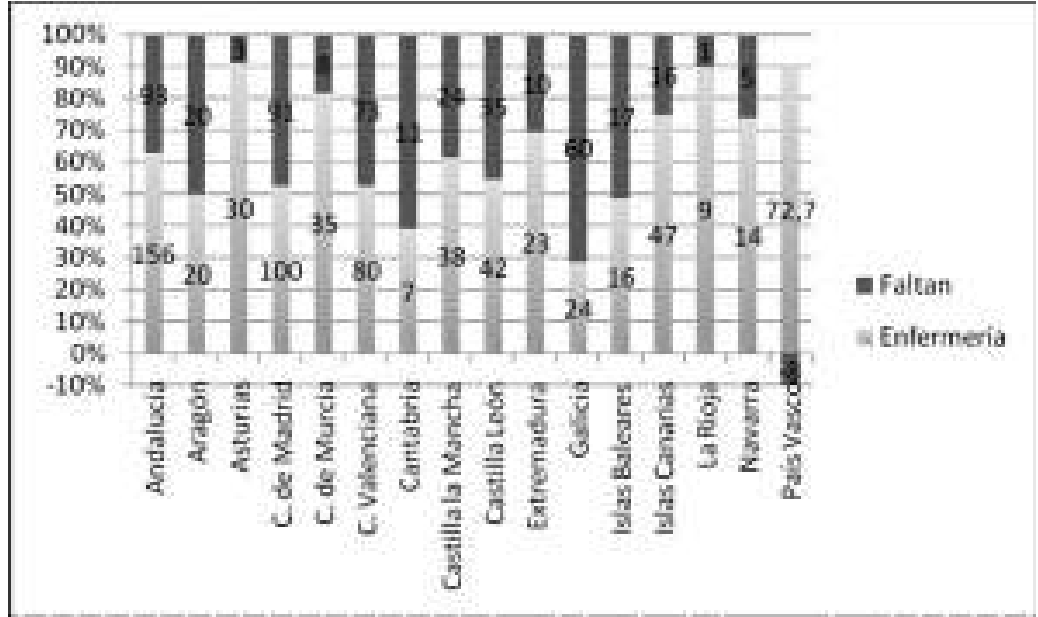

\section{Recursos de la red asistencial a la salud mental}

El Observatorio de Salud Mental de la AEN también nos aporta información acerca de dispositivos específicos para la atención de pacientes con trastorno mental grave (TMG). Algunos de estos dispositivos son los hospitales de día, las unidades de rehabilitación psicosocial, las unidades de media estancia y los dispositivos residenciales tipo miniresidencia o casa hogar. Veamos la dotación de plazas para tales dispositivos en las diferentes comunidades autónomas.

\section{Unidades de hospitalización parcial de adultos}

El criterio de calidad de la AEN para unidades de unidades de hospitalización parcial de adultos (hospitales de día de adultos) es de 10 plazas por cada 100.000 habitantes. Como podemos apreciar en la figura 7, la dotación de plazas en hospitales de día es muy desigual en las diferentes comunidades autónomas. Unicamente el Pais Vasco alcanza y supera este criterio de calidad. Cantabria, la Comunidad Valenciana, Aragón, Andalucía, Castilla la Mancha y Galicia no alcanzan siquiera el 50\% del criterio de calidad. Asturias, Canarias y la Rioja no han declarado ninguna plaza para este tipo de dispositivo. La Comunidad de Murcia y Extremadura no han aportado información al respecto. 


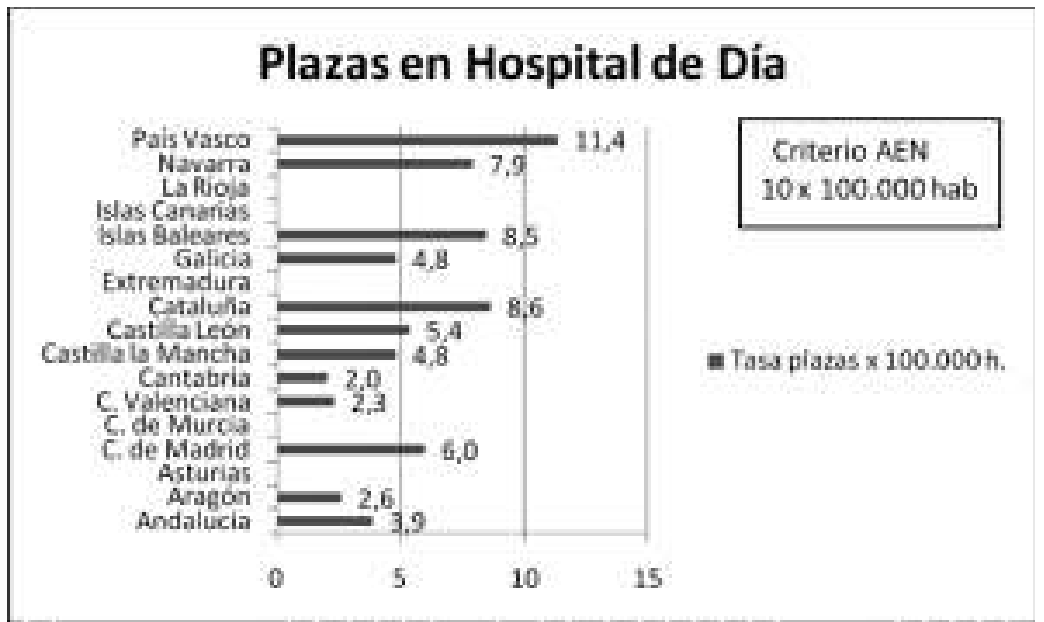

Centros de rehabilitación psicosocial

El criterio de calidad de la AEN para centros de rehabilitación psicosocial es de 20 plazas por cada 100.000 habitantes. Como podemos apreciar en la figura 8 , la dotación de plazas en centros de rehabilitación psicosocial es muy desigual en las diferentes comunidades autónomas. Sin embargo, para este tipo de dispositivos, dos terceras partes de las comunidades alcanzan y superan este criterio de calidad. Algunas comunidades como Castilla la Mancha, País Vasco, Cantabria, Aragón y Navarra, incluso duplican este criterio. Sin embargo otras comunidades como Andalucía, La Rioja, o Castilla y León, no alcanzan el 50 \% del criterio de calidad.

\section{Unidades hospitalarias de media estancia}

El criterio de calidad de la AEN para unidades de unidades de hospitalarias de rehabilitación de media estancia es de 8 plazas por cada 100.000 habitantes. Como podemos apreciar en la figura 9, aún cuando la dotación de plazas de rehabilitación de media estancia es desigual en las diferentes comunidades autónomas, la mayoría de ellas alcanza el criterio de calidad. La Comunidad Valenciana, Comunidad de Madrid, Andalucía, Castilla la Mancha y Cataluña no alcanzan el criterio de calidad. Algunas comunidades como Cantabria y Galicia duplican el criterio de calidad y Navarra llega a sextuplicarlo. Extremadura no aporta información al respecto.

Alternativas residenciales: miniresidencias.

El criterio de calidad de la AEN para las alternativas residenciales tipo miniresidencia o casa hogar es de 20 plazas por cada 100.000 habitantes. Como pode- 


\section{INFORMES}

Figura 8

Centros de Rehabilitación psicosocial. Plazas por cada 100.000 habitantes

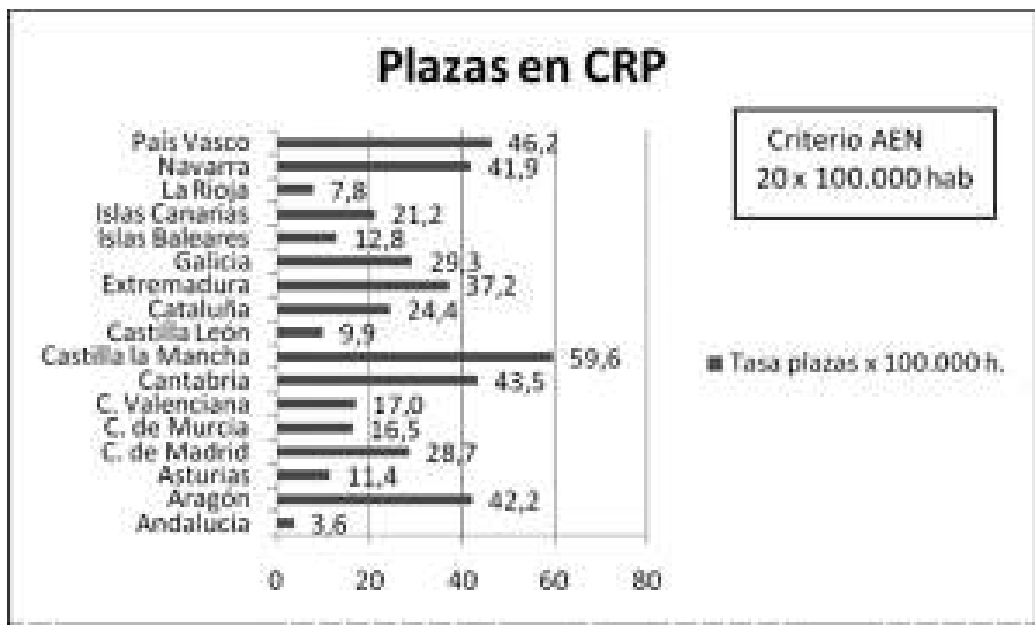

Figura 9

Unidades de Media Estancia. Plazas por cada 100.000 habitantes

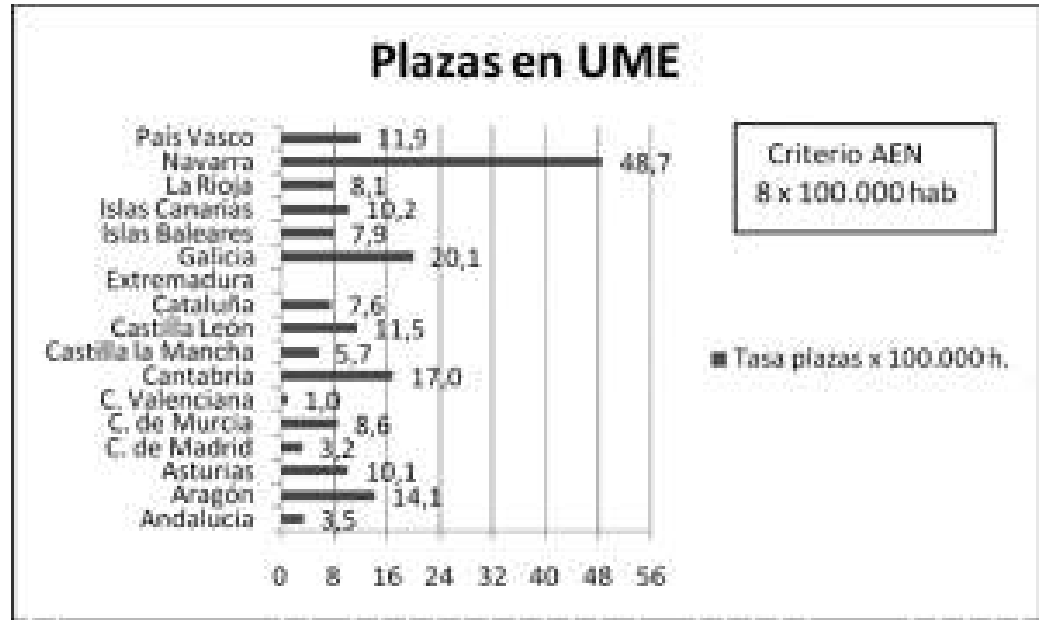

mos apreciar en la figura 10, la dotación de plazas de alternativas residenciales tipo miniresidencia es también muy desigual entre las diferentes comunidades autónomas y solamente la comunidad Navarra casi alcanza el criterio de calidad. Diez de las once comunidades que declaran tener plazas de miniresidencias no alcanzan siquiera el $50 \%$ del criterio de calidad, situándose la media en 4 plazas por cada 100.000 habitantes. La variabilidad es tal que hay comunidades que no cuentan 
siquiera con este tipo de dispositivos. Algunas comunidades como Asturias, Castilla y León y Cataluña no aportan datos. Otras comunidades como la Comunidad Valenciana, Murcia o La Rioja han declarado no disponer de ninguna plaza de este tipo. La Comunidad Valenciana, aunque dispone de plazas de residencia para pacientes con trastorno mental grave, éstas no se encuentran dotadas en dispositivos tipo casa hogar sino en grandes residencias.

Figura 10

Miniresidencias. Plazas por cada 100.000 habitantes

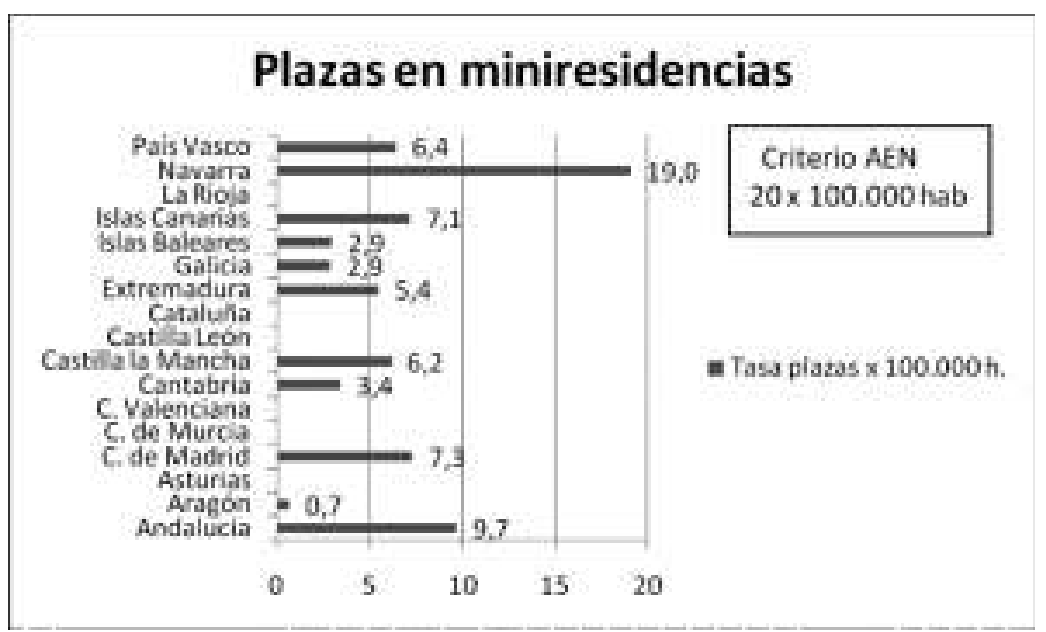

\section{Conclusiones}

Los recursos humanos y la red de dispositivos radicados en la comunidad, conforman el pilar de una asistencia a la salud mental basada en el modelo comunitario. Una adecuada dotación de profesionales y una amplia red de dispositivos comunitarios, suficientemente dotada de plazas para atender las necesidades presentadas por la población, particularmente por las personas con trastorno mental grave, es imprescindible para una atención de calidad en consonancia con las recomendaciones de documentos de consenso y guías de práctica clínica. La AEN, a partir de datos y documentos nacionales e internacionales, ha facilitado una serie de estimaciones de criterios de calidad asistencial, tanto para profesionales como para plazas en dispositivos asistenciales en función de ratios poblacionales. La combinación de estos criterios con los datos proporcionados por el Observatorio de Salud Mental de la AEN, permiten gestionar este conocimiento para conocer nuestra realidad asistencial en relación a los indicadores y poder tomar decisiones, relacionadas con la planificación asistencial, basadas en los datos. 
INFORMES

En este estudio hemos podido comprobar que la dotación de recursos humanos en los centros de salud mental de adultos es heterogénea y en general insuficiente en el conjunto de los servicios asistenciales de las comunidades autónomas. Faltan, ordenados por proporción de déficit entre los profesionales analizados, psicólogos clínicos, enfermería y psiquiatras. Los primeros apenas sobrepasan el $40 \%$ de las necesidades de profesionales, enfermería se sitúa en el entorno del $50 \%$ y la dotación de psiquiatras apenas llega al 70\% de las necesidades estimadas. De forma más concreta, los psiquiatras alcanzan sólo el $67 \%$ de las necesidades estimadas, calculándose el déficit en 539 nuevos psiquiatras. Únicamente País Vasco y Asturias superan el criterio de calidad. Galicia y las Islas Baleares son las comunidades autónomas con menor tasa y mayores necesidades. La dotación de enfermería supone el 54\% de las necesidades estimadas. Únicamente el País Vasco alcanza el criterio, aunque se acercan La Rioja y Asturias. Se estima que faltan 459 nuevas plazas de enfermería. Galicia y Cantabria son las comunidades autónomas con menor tasa y mayores necesidades. Los psicólogos clínicos únicamente alcanzan el $42 \%$ de las necesidades estimadas. Ninguna comunidad alcanza el criterio de calidad. Se estima que faltan 790 nuevos psicólogos. Galicia, Andalucía, País Vasco, Aragón, Madrid y Valencia son las comunidades con menor tasa y mayores necesidades. Una dotación suficiente y bien capacitada de estos profesionales, junto con otros como los trabajadores sociales, es básica para una atención real en la comunidad en base a programas comunitarios y no solo a dispositivos, como está siendo establecida en los documentos de consenso y guías de práctica clínica.

En cuanto a la existencia de dispositivos y plazas para la atención a los pacientes con trastorno mental grave, hemos analizado los datos de algunos de los más significativos. Los datos al respecto, proporcionados por el Observatorio de Salud Mental y en combinación con los criterios estimados por la AEN, en cuanto al número de número de plazas por población a atender, permite afirmar que aunque la mayoría de ellos están generalizados en la mayoría de las comunidades autónomas, la dotación de plazas es insuficiente para alcanzar unos estándares de calidad. Estas insuficiencias no se reparten por el igual en todo el estado, ni referidas a todos los dispositivos, siendo su dotación bastante heterogénea a través de las diferentes comunidades. Así en cuanto a hospitales de día, únicamente el País Vasco alcanza el criterio de plazas por ratio poblacional. Cantabria, la Comunidad Valenciana o Aragón son las comunidades con menor dotación de plazas. Respecto a los centros de rehabilitación psicosocial, 10 de las comunidades alcanzan o superan el criterio de calidad. Andalucía, La Rioja o Castilla y León no alcanzan a cubrir el 50\% de las plazas necesitadas. En cuanto a las unidades de media estancia, prácticamente 11 comunidades autónomas alcanzan o superan el criterio de calidad. La comunidad Valenciana, la Comunidad de Madrid y Andalucía no alcanzan a cubrir el $50 \%$ de las plazas necesitadas. Finalmente, las plazas de miniresidencias es muy 
insuficiente en la mayoría de las comunidades. Únicamente Navarra se acerca al estándar de calidad. Las demás comunidades no llegan ni a cubrir el $50 \%$ de las necesidades. Aragón, Galicia, Islas Baleares y Cantabria son las comunidades más infradotadas.

Junto a la escasez de recursos constatada, que afecta al derecho a la salud, la heterogeneidad entre comunidades en la dotación de los recursos humanos y de plazas en dispositivos de atención a las personas con trastorno mental grave, afecta al principio de equidad que debe regir en la prestación de la asistencia sanitaria en el conjunto del estado. El Observatorio de Salud Mental de la AEN y los indicadores de calidad son dos instrumentos que en combinación pueden permitir una evaluación y planificación asistencial desde criterios de calidad y que, a su vez, responda a al principio de equidad en el conjunto del estado. Para ello es básico que ambos instrumentos continúen siendo actualizados y perfeccionados para responder cada vez más adecuadamente a este objetivo. En este sentido se hace imprescindible, para garantizar el rigor y la exhaustividad en la recogida de datos del Observatorio, la colaboración de todas administraciones autonómicas en cuanto a la facilitación de todos los datos requeridos.

BIBLIOGRAFÍA:

(1) Salvador Sánchez, I. (coord.) El Observatorio de salud mental: análisis de los recursos de salud mental en España. Revista de la Asociación Española de Neuropsiquiatría, 2005, No monográfico 93, 1-85. Disponible en http://www.observatorio-aen.es/es/informes/observatorio-de-salud-mental-de-laasociaci-n-espa/observatorio-salud-mental-2003/download.html

(2) Salvador Sánchez, I. (coord.) El Observatorio de salud mental de la Asociación Española de Neuropsiquiatría. Análisis de la situación de la atención a la salud mental en las Comunidades Autónomas a diciembre de 2005. Cuadernos Técnicos, 7. Madrid: AEN, 2007. Disponible en http://www.observatorioaen.es/es/informes/observatorio-de-salud-mental-de-la-asociaci-n-espa/observatorio-salud-mental-2005/ download.html

(3) Jiménez Estévez, JF. (coord.). Observatorio de la Asociación Española de Neuropsiquiatría 2007. Web del Observatorio de Salud Mental de la AEN. Disponible en http://www.observatorio-aen.es .

(4) Jiménez Estévez, JF. (coord.). Observatorio de la Asociación Española de Neuropsiquiatría 2010. Web del Observatorio de Salud Mental de la AEN. Disponible en http://www.observatorio-aen.es .

(5) Jiménez Estévez, JF. (coord.) Observatorio de Salud Mental 2010 de la Asociación Española de Neuropsiquiatría. Informe descriptivo de la situación de la atención a la salud mental en las comunidades autónomas. Cuadernos Técnicos, 15. Madrid: AEN, 2011. Disponible en http://www.observatorioaen.es/es/informes/observatorio-de-salud-mental-de-la-asociaci-n-espa/observatorio-salud-mental-2010/ download.html

(6) Estrategia en Salud Mental del Sistema Nacional de Salud 2006. Ministerio de Sanidad y Consumo, 2007. Disponible en http://www.msps.es/organizacion/sns/planCalidadSNS/pdf/excelencia/salud_mental/ESTRATEGIA_SALUD_MENTAL_SNS_PAG_WEB.pdf 
INFORMES

(7) Declaración Europea de Salud Mental del Plan de Acción de Helsinki. Disponible en: http://www.msps.es/organizacion/sns/planCalidadSNS/pdf/excelencia/salud_mental/opsc_est17.pdf.pdf

(8) Estrategia en Salud Mental Sistema Nacional de Salud 2009-2013. Ministerio de Sanidad, Política Social e Igualdad, 2011. Disponible en http://www.msps.es/organizacion/sns/planCalidadSNS/ docs/saludmental/SaludMental2009-2013.pdf

(9) Bravo Ortíz, MF. (coord.) Hacia una atención comunitaria de Salud Mental de calidad. Cuadernos Técnicos, 4. Madrid: AEN, 2000. Disponible en http://www.aen.es/index.php?option=com_ docman\&task=doc_download $\&$ gid=125\&Itemid=119 